\title{
Fever as an unusual presentation of a common condition
}

\author{
J M M Gardner-Medwin, K L Lim, R J Powell
}

\section{Case Report}

The patient presented with a twelve month history of fever, lethargy and fatigue, superficial thrombophlebitis, myalgia/arthralgia with cramps in the left calf, tender nodules around her elbows, a widespread livido reticularis rash, pleuritic chest pain with shortness of breath on exertion and tingling in the peripheries. Her past history included long-standing photosensitivity, possible Raynaud's phenomenon, diffuse hair loss, five pregnancies including three spontaneous abortions in her first three pregnancies at eight, 12, and 26 weeks respectively, and a post partum deep venous thrombosis. Despite the normal/negative investigations shown in the table, which included an ESR, CRP, ANA, ANCA, and needle quadriceps muscle biopsy, she was diagnosed as having a systemic vasculitis, possibly systemic lupus erythematosus (SLE). She was started on oral prednisolone and intravenous pulse cyclophosphamide. Three days after starting therapy she developed a swollen tender left calf. Venogram confirmed an extensive ileo-femoral deep vein thrombosis, and she was anticoagulated with warfarin.

Despite two months of immunosuppressive therapy, she continued to be debilitated. In addition, she became anxious and increasingly short of breath on exertion with a resting tachycardia which resulted in the referral to Nottingham. On examination a prominent livido rash, multiple non-tender subcutaneous nodules around her elbows, and purpuric lesions on her lower limbs were documented. Chest auscultation revealed fine crackles at the right base. Cardiovascular examination was unremarkable, in particular no cardiac murmers were detected. During this admission, the spiking fever (fig 1) associated with a tachycardia was confirmed. She was taking 10 $\mathrm{mg}$ of prednisolone, having completed anticoagulation two weeks earlier.

A rising ESR and neutrophil count were noted (see table), a repeat muscle biopsy revealed type 2 atrophy, and a subcutaneous nodule was histologically demonstrated to be a lipoma. Other investigations are listed in the table. A chest radiograph revealed multiple areas of atelectasis with reticulonodular shadowing. Pulmonary function tests showed a restrictive defect, with reduced transfer

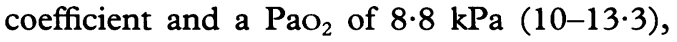
$\mathrm{PaCO}_{2} 4 \cdot 34 \mathrm{kPa}(4 \cdot 8-6 \cdot 1)$ on arterial blood gas analyses. Although multiple pulmonary emboli were suspected, a transbronchial biopsy was performed to exclude an atypical chest infection secondary to immunosuppressive therapy and a lymphoproliferative disorder. Atelectasis and a pulmonary artery thrombus were demonstrated on computed tomography

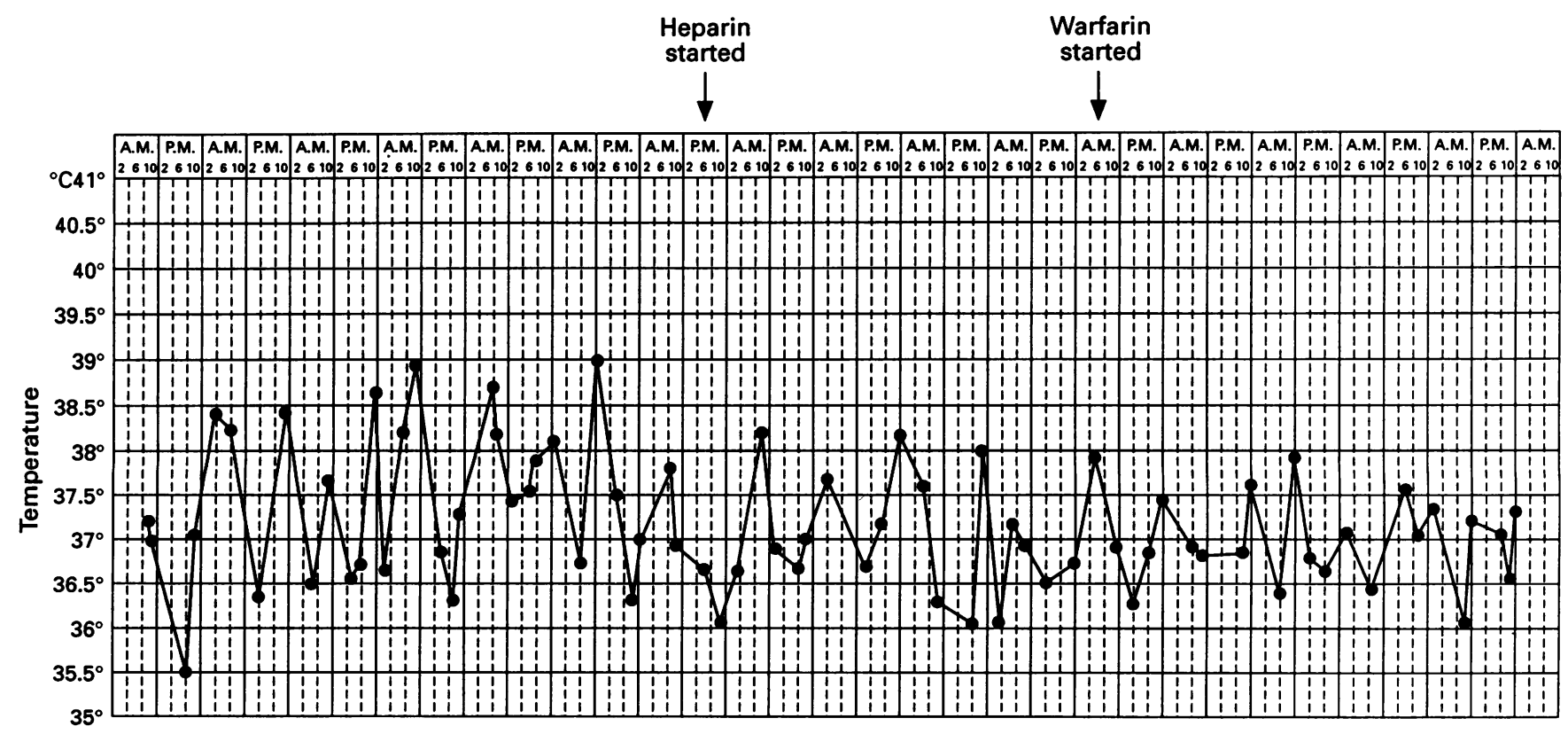

Figure 1 The patient's temperature chart and the start of anticoagulation. 
Investigations carried out

\begin{tabular}{|c|c|c|}
\hline Investigation & At first presentation & In Nottingham \\
\hline Neutrophil count $\left(\times 10^{9} / 1\right)$ & $5 \cdot 0$ & $\begin{array}{l}\text { Pre-warfarin serial } \\
\text { values } \\
1: 4 \cdot 56 \\
2: 8 \cdot 09 \\
3: 10 \cdot 1 \\
\text { Post-warfarin: } 5.95\end{array}$ \\
\hline $\operatorname{ESR}(\mathrm{mm} / \mathrm{h})$ & 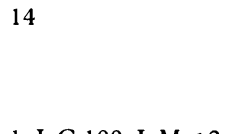 & $\begin{array}{l}\text { Pre-warfarin serial } \\
\text { values } \\
1: 41 \\
2: 69 \\
\text { Post-warfarin: } 22\end{array}$ \\
\hline ANA & $\begin{array}{l}\text { 1: } \operatorname{IgG} 100, \operatorname{Ig} M<2 \\
\text { 2: Negative }\end{array}$ & $\begin{array}{l}\text { Negative on two } \\
\text { occasions }\end{array}$ \\
\hline $\begin{array}{l}\text { ENA, anti-dsDNA, ANCA, aPL } \\
\text { C reactive protein } \\
\text { Complement } \\
\text { (C3, C4, C3dg) }\end{array}$ & $\begin{array}{l}\text { Negative } \\
\text { Not increased } \\
\text { Normal }\end{array}$ & $\begin{array}{l}\text { Negative } \\
\text { Not increased } \\
\text { Normal }\end{array}$ \\
\hline $\begin{array}{l}\text { Blood, urine and sputum cultures } \\
\text { Lupus anticoagulant }\end{array}$ & Not detected & $\begin{array}{l}\text { Repeatedly negative } \\
\text { Not detected }\end{array}$ \\
\hline INR stabilised on warfarin & $1 \cdot 6$ & $2 \cdot 6$ \\
\hline
\end{tabular}

$\mathrm{ESR}=$ Erythrocyte sedimentation rate; $\mathrm{ANA}=$ antinuclear antibody; ENA = extractable nuclear antigen; anti-dsDNA = antibody to double stranded DNA; ANCA $=$ antineutrophil cytoplasmic antibodies; aPL = antiphospholipid; INR = international normalised ratio.

and a ventilation perfusion scan confirmed multiple pulmonary emboli (fig 2). Warfarin was restarted with subsequent gradual resolution of her symptoms, in particular the fever. The raised ESR and neutrophilia likewise gradually returned to normal values.

\section{Discussion}

Fever and peripheral leucocytosis are recognised features of pulmonary emboli particularly where there is extensive pulmonary infarction, ${ }^{1}$ and recurrent pulmonary embolism can present insidiously as a pyrexia of unknown origin. In two cohorts each comprising approximately 100 patients with a documented fever greater than $38.5^{\circ} \mathrm{C}$ of unknown origin, persisting for more than seven days, pulmonary emboli were the cause in $1 \%$ and $3 \%$ of cases respectively. ${ }^{2}$
The history presented here with the emphasis on respiratory involvement, and the chest radiograph findings of atelectasis, all point to the diagnosis of pulmonary emboli. However, the apparent multisystem involvement at her initial presentation, despite the lack of histological and immunological data, led to the clinical diagnosis of vasculitis. The diagnosis of SLE was suggested on the basis of fever, diffuse alopecia, myalgia/arthralgia, and pleuritic chest pain, associated with a weakly positive ANA. However, the significance of an ANA titre of 100 or less in a 61 year old woman is doubtful, contrasting with an ANA titre of 50 in a young woman which may have major clinical importance. Low titred ANAs become increasingly common in females with advancing age. Subsequent testing for ANA in this patient was repeatedly negative. Although the existence of 'ANA negative' SLE is accepted, it is uncommon, and is usually characterised by cutaneous involvement. Anti-Ro antibodies may often be present in these ANA negative cases, and should be specifically looked for as the anti-Ro reactivity will not be recognised by standard antinuclear antibody immunofluorescence techniques. ${ }^{3}$ Anti-neutrophil cytoplasmic antibodies (ANCA) can be valuable in the investigation of a patient with suspected vasculitis, however, ANCAs have a higher specificity but a lower sensitivity than ANAs for their respective diseases, so that a negative ANCA does not exclude small vessel vasculitis, but a negative ANA in the absence of skin involvement makes SLE highly unlikely. It is possible for the unwary to miss pANCA in the presence of a high titre of ANA. However formalin fixation prevents the perinuclear aggregation of the relevant antigens, and thus a cytoplasmic pattern is seen, allowing easier identification of ANCA. Subsequent solid

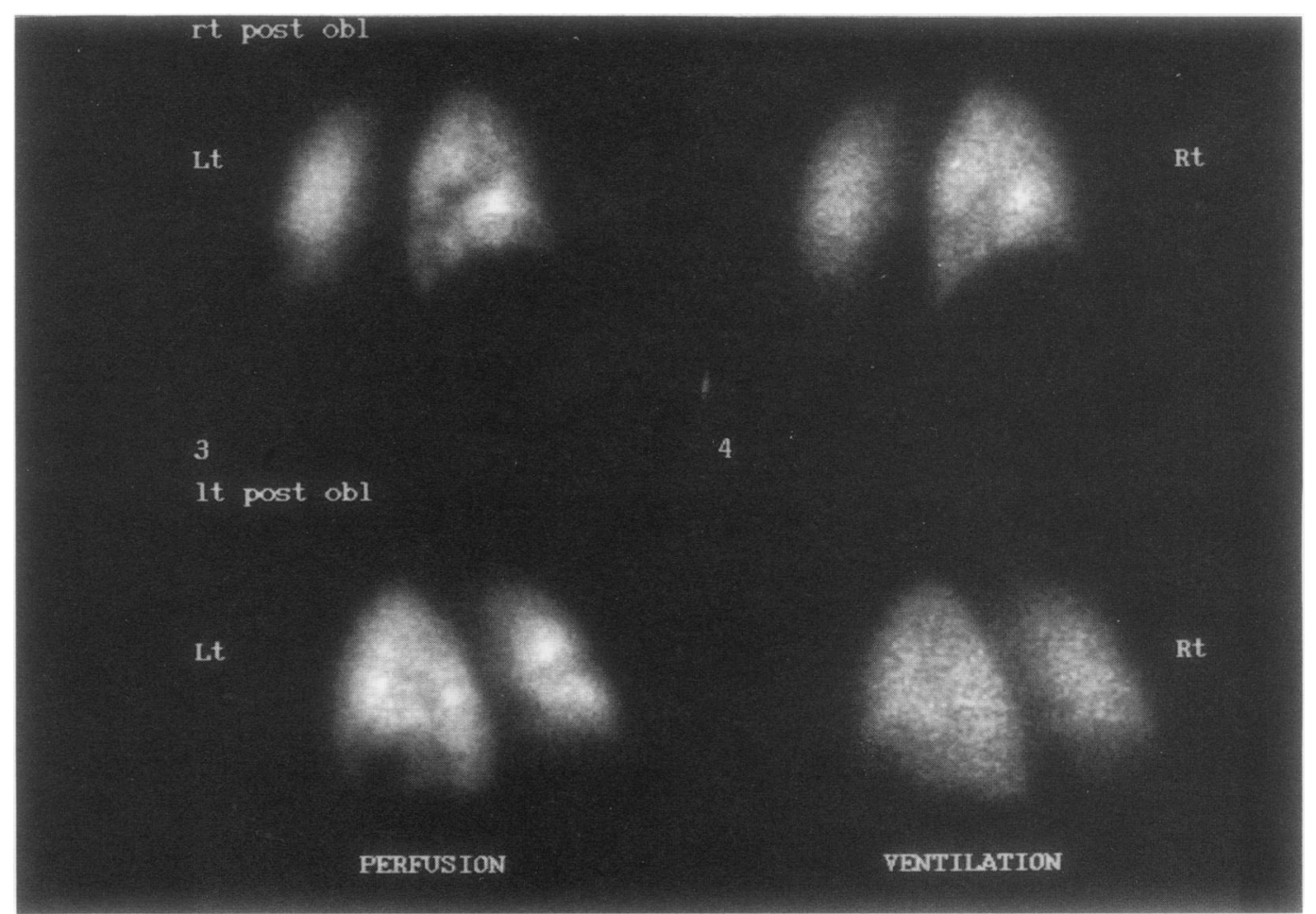

Figure 2 The ventilation and perfusion mismatches on scan. 
phase analysis of ANCA may be appropriate in such instances.

The antiphospholipid antibody syndrome is relevant to this case, however, both antiphospholipid antibodies and the lupus anticoagulant remained negative on repeated testing. Other important differential diagnoses were subacute bacterial endocarditis, and adult-onset Still's disease. The former was excluded by repeated negative blood cultures and the absence of a cardiac murmur on regular auscultation, whilst the latter was thought unlikely as clinically the rash was classical livido reticularis.

Further interesting issues arising from this case include the troublesome paraesthesiae, which blood gas measurement confirmed were due to hyperventilation, and the lack of an ESR response at first presentation, despite the long history of fever. The later rise in ESR and neutrophil count may reflect the subsequent extent of lung infarction and inflammation. The apparent lack of response to warfarin at the first hospital may reflect inadequate anticoagulation, as shown by the INR in the table, whereas in Nottingham the INR was maintained within the therapeutic range.
In summary, the lack of serological and histological confirmation of a systemic vasculitis and the absence of a response to high dose oral steroid therapy, made the diagnosis of vasculitis unlikely and a complete reevaluation of the case was required. This case highlights the need to consider the relative weighting of investigations in the diagnosis of suspected vasculitis, and the difficulties caused by the pursuit of the diagnosis of vasculitis without the exclusion of common conditions, such as thromboembolic disease.

\section{The lesson}

- Multiple pulmonary emboli should be considered in a patient with fever and progressive dyspnoea.

1 Spencer H, ed. Pathology of the Lung, 4th ed. Pergamon

2 Larson E, Featherstone H J, Petersdorf R. Fever of undetermined origin: diagnosis and follow up of 105 cases, 1970-1980. Medicine 1982; 61 (5): 269-92

3 Shur P H. Clinical features of SLE. In: Kelley W N, Harris E D, Ruddy S, Sledge C B, eds. Textbook of rheumatology, 4th ed. Philadelphia: W B Saunders, 1993: 1017-42. 